\title{
An optimal portfolio, consumption-leisure and retirement choice problem with CES utility: a dynamic programming approach
}

Ho-Seok Lee ${ }^{1}$ and Yong Hyun Shin ${ }^{2 *}$ (D)

"Correspondence:

yhshin@sookmyung.ac.kr

${ }^{2}$ Department of Mathematics,

Sookmyung Women's University,

Seoul, 04310, Republic of Korea

Full list of author information is

available at the end of the article

\section{算 Springer}

\begin{abstract}
In this paper, we study an optimal portfolio, consumption-leisure and retirement choice problem for an infinitely lived economic agent with a CES utility function. Using the dynamic programming method, we obtain the value function and optimal investment, consumption, leisure, and retirement strategies in analytic form. Numerically we observe that the threshold retirement wealth level is an increasing function with respect to the elasticity of substitution.
\end{abstract}

MSC: Primary 91G10; secondary 91G80

Keywords: consumption and leisure; portfolio selection; voluntary retirement; CES utility; dynamic programming method; free boundary value problem

\section{Introduction}

We consider an optimal portfolio and consumption-leisure choice problem of an infinitely lived economic agent with a voluntary retirement option and a CES utility function of consumption and leisure. The agent can choose her labor supply flexibly above a certain minimum level in accordance with the trade-off between utility from leisure and labor income. The economic agent receives labor income proportional to the amount of labor supplied before retirement and enjoys full leisure after retirement at the cost of labor income.

Bodie et al. [1] investigated the influence of the labor supply flexibility on portfolio and consumption choice under the lifetime portfolio and consumption choice model of Merton [2,3]. Farhi and Panageas [4] studied the optimal portfolio, consumption and retirement choice problem of an economic agent with a Cobb-Douglas utility function and a binomial leisure rate process ( $l_{1}$ before retirement and $\bar{l}$ after retirement) using the martingale method. Shin [5] solved the problem using the dynamic programming method, and Koo et al. [6] extended the model by allowing the leisure rate process to be chosen flexibly, also using the dynamic programming method. Borrowing constraints have significant effects on an economic agent's optimal portfolio, consumption and retirement choice, and this is well documented in the literature, for example, Dybvig and Liu [7]. Barucci and Marazzina [8] also considered borrowing constraints with stochastic labor income in solving optimal consumption, investment, labor supply and retirement choice problem through a duality approach. Choi et al. [9] extended Farhi and Panageas [4] by

(c) 2015 Lee and Shin. This article is distributed under the terms of the Creative Commons Attribution 4.0 International License (http://creativecommons.org/licenses/by/4.0/), which permits unrestricted use, distribution, and reproduction in any medium, provided you give appropriate credit to the original author(s) and the source, provide a link to the Creative Commons license, and indicate if changes were made. 
employing a general CES utility function, which has the Cobb-Douglas utility function as a special case, and by allowing a continuum between labor and leisure. In Choi et al. [9], the martingale method is used to provide an analytic form for the value function and optimal strategies.

We revisit the optimization problem of Choi et al. [9] to give some methodological contributions and supply some numerical results which were not considered therein. We use the dynamic programming approach based on Karatzas et al. [10] to derive the closedform solutions including the value function and optimal strategies. Since our optimization problem utilizes CES utility, it is important to investigate the effect of the elasticity of substitution between consumption and leisure on the optimal policies. Numerically we observe that the threshold retirement wealth level is an increasing function with respect to the elasticity of substitution. This is due to the fact that an economic agent with a large elasticity of substitution between consumption and leisure may consume more and enjoy higher utility than an economic agent with a small elasticity of substitution when the optimal leisure rate reaches the maximum leisure rate allowed while working. We also show that our results converge to those with Cobb-Douglas utility in Koo et al. [6] as the elasticity of substitution goes to 1 .

The rest of the paper is organized as follows. Section 2 sets up the economic model. In Section 3, we solve the optimization problem using the dynamic programming method. Section 4 provides numerical examples with a limiting case, and Section 5 concludes.

\section{The economy}

In the financial market, we assume that the agent has investment opportunities given by a riskless asset with a constant interest rate $r>0$, and one risky asset $S_{t}$ whose price evolves according to the following stochastic differential equation: $d S_{t} / S_{t}=\mu d t+\sigma d B_{t}$, where $\mu$ is a constant mean rate of return, $\sigma$ is a constant volatility, and $B_{t}$ is a standard Brownian motion on a probability space $(\Omega, \mathcal{F}, \mathbb{P}) .\left\{\mathcal{F}_{t}\right\}_{t \geq 0}$ is the $\mathbb{P}$-augmentation of the filtration generated by the standard Brownian motion $\left\{B_{t}\right\}_{t \geq 0}$.

Let $\pi_{t}$ be the amount of money invested in the risky asset at time $t, c_{t} \geq 0$ be the agent's consumption rate process at time $t$, and $l_{t} \geq 0$ be the agent's leisure rate process at time $t$. $\pi_{t}, c_{t}$, and $l_{t}$ are all $\mathcal{F}_{t}$-progressively measurable and satisfy the following technical conditions:

$$
\int_{0}^{t} \pi_{s}^{2} d s<\infty, \quad \int_{0}^{t} c_{s} d s<\infty \quad \text { and } \quad \int_{0}^{t} l_{s} d s<\infty \quad \text { for all } t \geq 0 \text { a.s. }
$$

Let $\tau$ be the (voluntary) retirement time from labor which will be considered as an optimal stopping time. The assumptions for the leisure rate process $l_{t}$ are given by

$$
0 \leq l_{t} \leq L<\bar{L} \quad \text { for } 0 \leq t<\tau \quad \text { and } \quad l_{t}=\bar{L} \quad \text { for } t \geq \tau
$$

where $L, \bar{L}$ are constants (see Choi et al. [9] and Koo et al. [6]). Let $w$ be the constant wage rate. Then $w\left(\bar{L}-l_{t}\right)>0$ is the labor income rate at time $t(<\tau)$ resulting from the laborleisure choice before retirement. However, since we choose $l_{t}=\bar{L}$ at time $t(\geq \tau)$, there is no labor income after retirement, that is, $w\left(\bar{L}-l_{t}\right) \equiv 0$. Therefore the agent's wealth process $x_{t}$ at time $t$, with a given initial endowment $x_{0}=x$, evolves according to the following 
stochastic differential equation:

$$
d x_{t}=\left[r x_{t}+\pi_{t}(\mu-r)-c_{t}+w\left(\bar{L}-l_{t}\right)\right] d t+\sigma \pi_{t} d B_{t} .
$$

A quadruple $(\mathbf{c}, \boldsymbol{\pi}, \mathbf{l}, \tau)$ is called admissible at initial capital $x_{0}=x>-w \bar{L} / r$ if $x_{t}>-w \bar{L} / r$ and $0 \leq l_{t} \leq L$ for $0 \leq t<\tau$, and $x_{t} \geq 0$ for $t \geq \tau$, where $\mathbf{c} \triangleq\left\{c_{t}\right\}_{t \geq 0}, \pi \triangleq\left\{\pi_{t}\right\}_{t \geq 0}$, and $\mathbf{l} \triangleq\left\{l_{t}\right\}_{t \geq 0}$. Here $w \bar{L} / r$ can be considered as the present value of the (maximum) future labor income of the agent.

\section{The optimization problem}

In this paper, we assume that the utility function $u(\cdot, \cdot)$ is of the CES type as follows:

$$
u(c, l) \triangleq \frac{1}{\alpha} \cdot \frac{\left\{\alpha c^{\rho}+(1-\alpha) l^{\rho}\right\}^{\frac{1-\gamma}{\rho}}}{1-\gamma}, \quad \rho<1, \rho \neq 0,0<\alpha<1, \gamma>0 \text { and } \gamma \neq 1 \text {, }
$$

where $\gamma$ is the agent's coefficient of relative risk aversion for consumption and leisure, $\alpha$ is a measurement of consumption's contribution to the agent's utility, and $1 /(1-\rho)$ is the elasticity of substitution between consumption and leisure.

The agent's optimization problem is to maximize the following expected discounted utility:

$$
J(x ; \mathbf{c}, \boldsymbol{\pi}, \mathbf{l}, \tau) \triangleq \mathbb{E}\left[\int_{0}^{\tau} e^{-\beta t} u\left(c_{t}, l_{t}\right) d t+e^{-\beta \tau} U\left(x_{\tau}\right)\right],
$$

where $\beta>0$ is the subjective discount rate and

$$
U(x)=\max _{(\mathbf{c}, \boldsymbol{\pi})} \mathbb{E}\left[\int_{0}^{\infty} e^{-\beta t} u\left(c_{t}, \bar{L}\right) d t\right]
$$

Thus the agent's optimization problem is given by

$$
V(x) \triangleq \max _{(\mathbf{c}, \boldsymbol{I}, \mathbf{l}, \tau) \in \mathcal{A}(x)} J(x ; \mathbf{c}, \boldsymbol{\pi}, \mathbf{l}, \tau)
$$

where $\mathcal{A}(x)$ is the set of all admissible quadruples at $x>0$.

Remark 3.1 For later use, we define a quadratic algebraic equation as follows:

$$
g(n) \triangleq \frac{1}{2} \theta^{2} n^{2}+\left(\beta-r+\frac{1}{2} \theta^{2}\right) n-r=0,
$$

where $\theta \triangleq(\mu-r) / \sigma$ is the market price of the risk. Because $g(0)<0$ and $g(-1)<0$, it follows that the quadratic equation $g(n)=0$ has two real roots $n_{1}$ and $n_{2}$ satisfying $n_{1}>0$ and $n_{2}<-1$. Also, we define $p_{1} \triangleq n_{1}+1$ and $p_{2} \triangleq n_{2}+1$.

Now we provide the following assumption that holds throughout the paper and guarantees the optimization problem (3.3) will be well defined. 
Assumption 3.1 For $u_{L}(c) \triangleq u(c, L)$ and $u_{\bar{L}}(c) \triangleq u(c, \bar{L})$, we assume that

$$
\int_{c}^{\infty} \frac{d y}{\left(u_{L}^{\prime}(y)\right)^{n_{2}}}<\infty \text { and } \int_{c}^{\infty} \frac{d y}{\left(u_{\bar{L}}^{\prime}(y)\right)^{n_{2}}}<\infty \quad \text { for any } c>0 .
$$

Before retirement $(t<\tau)$, we derive the following Hamilton-Jacobi-Bellman (HJB) equation for the value function $V(x)$ by using the dynamic programming principle:

$$
\beta V(x)=\max _{c, \pi, l \leq L}\left[\{r x+\pi(\mu-r)-c+w(\bar{L}-l)\} V^{\prime}(x)+\frac{1}{2} \sigma^{2} \pi^{2} V^{\prime \prime}(x)+u(c, l)\right] .
$$

We postulate that there exists a threshold wealth level $\bar{x}$ (see, for similar conjecture and validation, Choi and Shim [11] or Dybvig and Liu [12]) corresponding to the optimal retirement time $\tau^{*}$ such that the value function $V(x)$ satisfies HJB equation (3.4) for $x<\bar{x}$ and $V(x)=U(x)$ in (3.2) for $x \geq \bar{x}$.

Theorem 3.1 Assume that a strictly increasing function $v(x) \in C^{1}(-w \bar{L} / r, \infty)$ solves HJB equation (3.4) for $x<\bar{x}$ and $v(x)=U(x)$ in (3.2) for $x \geq \bar{x}$, where $\bar{x}$ is determined by the smooth-pasting (or continuous differentiability) condition at $x=\bar{x}$. Also assume that $v(x) \in$ $C^{2}(-w \bar{L} / r, \infty) \backslash\{\bar{x}\}$. Then $v(x) \geq J(x ; \mathbf{c}, \boldsymbol{\pi}, \mathbf{l}, \tau)$ for any admissible control $(\mathbf{c}, \boldsymbol{\pi}, \mathbf{l}, \tau) \in \mathcal{A}(x)$.

Let us define

$$
\begin{array}{ll}
\tau^{*} \triangleq \inf \left\{t \geq 0: x_{t} \geq \bar{x}\right\}, & \\
c_{t}^{*} \triangleq c_{t}^{1, *}, \quad \pi_{t}^{*} \triangleq \pi_{t}^{1, *}, & l_{t}^{*} \triangleq l_{t}^{1, *} \quad \text { for } 0 \leq t<\tau^{*}, \\
c_{t}^{*} \triangleq c_{t}^{2, *}, \quad \pi_{t}^{*} \triangleq \pi_{t}^{2, *}, & l_{t}^{*} \triangleq l_{t}^{2, *} \quad \text { for } t \geq \tau^{*},
\end{array}
$$

where $\left(c_{t}^{1, *}, \pi_{t}^{1, *}, l_{t}^{1, *}\right)$ is the maximizer of HJB equation (3.4) and $\left(c_{t}^{2, *}, \pi_{t}^{2, *}, l_{t}^{2, *}\right)$ is the optimal consumption, portfolio, and leisure process corresponding to the post retirement value function $U(x)$. Then

$$
v(x)=V(x)=\max _{(\mathbf{c}, \boldsymbol{\pi}, \mathbf{l}, \tau) \in \mathcal{A}(x)} J(x ; \mathbf{c}, \boldsymbol{\pi}, \mathbf{l}, \tau)=J\left(x ; \mathbf{c}^{*}, \boldsymbol{\pi}^{*}, \mathbf{l}^{*}, \tau^{*}\right) .
$$

Proof Let us define a function $W(\cdot, \cdot)$ as follows:

$$
W\left(t, x_{t}\right)=e^{-\beta t} v\left(x_{t}\right)
$$

Applying Itô's rule to $W\left(t, x_{t}\right)$ gives us

$$
\begin{aligned}
d W\left(t, x_{t}\right)= & e^{-\beta t}\left[\left\{r x_{t}+\pi_{t}(\mu-r)-c_{t}+w\left(\bar{L}-l_{t}\right)\right\} v^{\prime}\left(x_{t}\right)+\frac{1}{2} \sigma^{2} \pi_{t}^{2} v^{\prime \prime}\left(x_{t}\right)-\beta v\left(x_{t}\right)\right] d t \\
& +\sigma \pi_{t} e^{-\beta t} v^{\prime}\left(x_{t}\right) d B_{t} \\
\leq & -e^{-\beta t} u\left(c_{t}, l_{t}\right) d t+\sigma \pi_{t} e^{-\beta t} v^{\prime}\left(x_{t}\right) d B_{t},
\end{aligned}
$$

for $0 \leq t<\tau^{*}$, where the inequality comes from HJB equation (3.4) and

$$
v\left(x_{0}\right) \geq \int_{0}^{s \wedge \tau^{*}} e^{-\beta t} u\left(c_{t}, l_{t}\right) d t+W\left(s \wedge \tau^{*}, x_{s \wedge \tau^{*}}\right)-\int_{0}^{s \wedge \tau^{*}} \sigma \pi_{t} e^{-\beta t} v^{\prime}\left(x_{t}\right) d B_{t}
$$


for any $s \geq 0$. Since the second integral of the right-hand side of (3.5) is a bounded local martingale and hence a martingale, we obtain

$$
v(x) \geq \mathbb{E}\left[\int_{0}^{s \wedge \tau^{*}} e^{-\beta t} u\left(c_{t}, l_{t}\right) d t+e^{-\beta\left(s \wedge \tau^{*}\right)} v\left(x_{s \wedge \tau^{*}}\right)\right]
$$

for any admissible control $\left(\mathbf{c}, \boldsymbol{\pi}, \mathbf{l}, \tau^{*}\right) \in \mathcal{A}(x)$. Letting $s \uparrow \infty$ and using the Lebesgue dominated convergence theorem, we have

$$
v(x) \geq \mathbb{E}\left[\int_{0}^{\tau^{*}} e^{-\beta t} u\left(c_{t}, l_{t}\right) d t+e^{-\beta \tau^{*}} U\left(x_{\tau^{*}}\right)\right]=J\left(x ; \mathbf{c}, \boldsymbol{\pi}, \mathbf{l}, \tau^{*}\right) .
$$

If $\left(c_{t}^{1, *}, \pi_{t}^{1, *}, l_{t}^{1, *}\right)$ is a maximizer of HJB equation (3.4), the inequality in (3.6) becomes equality and, consequently, $v(x)=V(x)$.

Theorem 3.2 The value function $V(x)$ of the optimization problem (3.3) is given by

$$
V(x)= \begin{cases}(\alpha+h)^{\frac{1-\gamma-\rho}{\rho}}\left\{\frac{n_{2}}{p_{2}} A_{2}(C(x))^{-\gamma n_{2}-\gamma}-\frac{\tilde{h}}{\gamma K}(C(x))^{1-\gamma}\right\} & \text { for }-\frac{w \bar{L}}{r}<x<\tilde{x}, \\ \frac{n_{1}}{p_{1}} B_{1}\left(u_{L}^{\prime}\left(C_{L}(x)\right)\right)^{p_{1}}+\frac{n_{2}}{p_{2}} B_{2}\left(u_{L}^{\prime}\left(C_{L}(x)\right)\right)^{p_{2}}+J_{L}\left(C_{L}(x)\right) & \text { for } \tilde{x} \leq x<\bar{x}, \\ \bar{J}(\bar{C}(x)) & \text { for } x \geq \bar{x},\end{cases}
$$

where $\tilde{x}$ is the threshold wealth level corresponding to the leisure rate of $l=L$, and $\bar{x}$ is the threshold wealth level corresponding to the optimal retirement time $\tau$, with $\tilde{x}<\bar{x}$. Let $\tilde{c}$ and $\bar{c}$ be the optimal consumption rates corresponding to $\tilde{x}$ and $\bar{x}$, respectively. $\tilde{c}, B_{1}, \bar{c}, \bar{x}, B_{2}, A_{2}$, and $\tilde{x}$ are given as follows:

$$
\begin{aligned}
\tilde{c}= & \left(\frac{w \alpha}{1-\alpha}\right)^{\frac{1}{1-\rho}} L, \\
B_{1}= & \frac{\tilde{h}}{\left(\frac{n_{2}}{p_{2}}-\frac{n_{1}}{p_{1}}\right) \gamma K}\left\{1-\frac{n_{2}}{p_{2}}(1-\gamma)\right\} \tilde{c}\left(u_{L}^{\prime}(\tilde{c})\right)^{-n_{1}} \\
& -\frac{n_{2}}{\left(\frac{n_{2}}{p_{2}}-\frac{n_{1}}{p_{1}}\right) p_{2}}\left\{\frac{w \bar{L}}{r}+X_{L, p}(\tilde{c})\right\}\left(u_{L}^{\prime}(\tilde{c})\right)^{-n_{1}}+\frac{J_{L}(\tilde{c})}{\frac{n_{2}}{p_{2}}-\frac{n_{1}}{p_{1}}}\left(u_{L}^{\prime}(\tilde{c})\right)^{-p_{1}},
\end{aligned}
$$

$\bar{c}$ can be determined from the following algebraic equation:

$$
\begin{aligned}
\left(\frac{n_{2}}{p_{2}}\right. & \left.-\frac{n_{1}}{p_{1}}\right) B_{1}\left(\frac{\bar{J}^{\prime}(\bar{c})}{\bar{X}^{\prime}(\bar{c})}\right)^{p_{1}}+\frac{n_{2}}{p_{2}} \frac{\bar{J}^{\prime}(\bar{c})}{\bar{X}^{\prime}(\bar{c})}\left\{X_{L, p}\left(I_{L}\left(\frac{\bar{J}^{\prime}(\bar{c})}{\bar{X}^{\prime}(\bar{c})}\right)\right)-\bar{X}(\bar{c})\right\} \\
& -J_{L}\left(I_{L}\left(\frac{\bar{J}^{\prime}(\bar{c})}{\bar{X}^{\prime}(\bar{c})}\right)\right)+\bar{J}(\bar{c})=0
\end{aligned}
$$

where

$$
\begin{aligned}
X_{L, p}(c)= & -\frac{2}{\theta^{2}\left(n_{1}-n_{2}\right)}\left\{\frac{\left(u_{L}^{\prime}(c)\right)^{n_{1}}}{n_{1}} \int_{0}^{c} \frac{d y}{\left(u_{L}^{\prime}(y)\right)^{n_{1}}}+\frac{\left(u_{L}^{\prime}(c)\right)^{n_{2}}}{n_{2}} \int_{c}^{\infty} \frac{d y}{\left(u_{L}^{\prime}(y)\right)^{n_{2}}}\right\} \\
& +\frac{c}{r}-\frac{w(\bar{L}-L)}{r},
\end{aligned}
$$




$$
\begin{aligned}
& J_{L}(c)=\frac{u_{L}(c)}{\beta}-\frac{2}{\theta^{2}\left(p_{1}-p_{2}\right)}\left\{\frac{\left(u_{L}^{\prime}(c)\right)^{p_{1}}}{p_{1}} \int_{0}^{c} \frac{d y}{\left(u_{L}^{\prime}(y)\right)^{n_{1}}}+\frac{\left(u_{L}^{\prime}(c)\right)^{p_{2}}}{p_{2}} \int_{c}^{\infty} \frac{d y}{\left(u_{L}^{\prime}(y)\right)^{n_{2}}}\right\}, \\
& \bar{X}(c)=\frac{c}{r}-\frac{2}{\theta^{2}\left(n_{1}-n_{2}\right)}\left\{\frac{\left(u_{\bar{L}}^{\prime}(c)\right)^{n_{1}}}{n_{1}} \int_{0}^{c} \frac{d y}{\left(u_{\bar{L}}^{\prime}(y)\right)^{n_{1}}}+\frac{\left(u_{\bar{L}}^{\prime}(c)\right)^{n_{2}}}{n_{2}} \int_{c}^{\infty} \frac{d y}{\left(u_{\bar{L}}^{\prime}(y)\right)^{n_{2}}}\right\} \\
& \bar{J}(c)=\frac{u_{\bar{L}}(c)}{\beta}-\frac{2}{\theta^{2}\left(p_{1}-p_{2}\right)}\left\{\frac{\left(u_{\bar{L}}^{\prime}(c)\right)^{p_{1}}}{p_{1}} \int_{0}^{c} \frac{d y}{\left(u_{\bar{L}}^{\prime}(y)\right)^{n_{1}}}+\frac{\left(u_{\bar{L}}^{\prime}(c)\right)^{p_{2}}}{p_{2}} \int_{c}^{\infty} \frac{d y}{\left(u_{\bar{L}}^{\prime}(y)\right)^{n_{2}}}\right\},
\end{aligned}
$$

and $I_{L}(\cdot)$ is the inverse function of $u_{L}^{\prime}(\cdot) \cdot \bar{x}$ is given by

$$
\bar{x}=\bar{X}(\bar{c})=\bar{X}(\bar{C}(\bar{x}))
$$

where $\bar{C}(\cdot)$ is the inverse function of $\bar{X}(\cdot)$. We then obtain $B_{2}$ as follows:

$$
B_{2}=-B_{1}\left(u_{L}^{\prime}\left(\bar{c}_{L}\right)\right)^{n_{1}-n_{2}}+\left\{\bar{x}-X_{L, p}\left(\bar{c}_{L}\right)\right\}\left(u_{L}^{\prime}\left(\bar{c}_{L}\right)\right)^{-n_{2}},
$$

where

$$
\bar{c}_{L}=I_{L}\left(\frac{\bar{J}^{\prime}(\bar{c})}{\bar{X}^{\prime}(\bar{c})}\right)
$$

and $A_{2}$ can be determined by

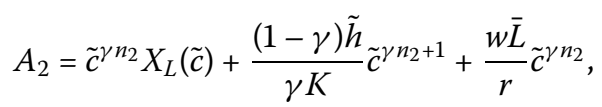

where

$$
X_{L}(c)=B_{1}\left(u_{L}^{\prime}(c)\right)^{n_{1}}+B_{2}\left(u_{L}^{\prime}(c)\right)^{n_{2}}+X_{L, p}(c)
$$

The threshold wealth level $\tilde{x}$ is given by

$$
\tilde{x}=X_{L}(\tilde{c}),
$$

where

$$
\begin{aligned}
& K \triangleq r+\frac{\beta-r}{\gamma}+\frac{\gamma-1}{2 \gamma^{2}} \theta^{2}, \quad \tilde{h} \triangleq-\frac{(\alpha+h) \gamma}{\alpha(1-\gamma)}, \quad h \triangleq(1-\alpha)\left(\frac{1-\alpha}{w \alpha}\right)^{\frac{\rho}{1-\rho}}, \\
& X(c)=A_{2} c^{-\gamma n_{2}}-\frac{(1-\gamma) \tilde{h}}{\gamma K} c-\frac{w \bar{L}}{r},
\end{aligned}
$$

$C(\cdot)$ is the inverse function of $X(\cdot)$, and $C_{L}(\cdot)$ is the inverse function of $X_{L}(\cdot)$.

Proof Due to the constraint $l_{t} \leq L$ for $t<\tau$, we introduce another wealth threshold level $\tilde{x}$ such that HJB equation (3.4) can be split into

$$
\beta V(x)=\left[\left\{r x+\pi^{*}(\mu-r)-c^{*}+w\left(\bar{L}-l^{*}\right)\right\} V^{\prime}(x)+\frac{1}{2} \sigma^{2}\left(\pi^{*}\right)^{2} V^{\prime \prime}(x)+u\left(c^{*}, l^{*}\right)\right]
$$


for $-w \bar{L} / r<x<\tilde{x}$, where

$$
\pi^{*}=-\frac{\theta}{\sigma} \frac{V^{\prime}(x)}{V^{\prime \prime}(x)}, \quad V^{\prime}(x)=(\alpha+h)^{\frac{1-\gamma-\rho}{\rho}}\left(c^{*}\right)^{-\gamma}, \quad l^{*}=\left(\frac{1-\alpha}{w \alpha}\right)^{\frac{1}{1-\rho}} c^{*}
$$

are obtained from the first order conditions (FOCs), and

$$
\beta V(x)=\left[\left\{r x+\pi^{*}(\mu-r)-c^{*}+w(\bar{L}-L)\right\} V^{\prime}(x)+\frac{1}{2} \sigma^{2}\left(\pi^{*}\right)^{2} V^{\prime \prime}(x)+u_{L}\left(c^{*}\right)\right]
$$

for $\tilde{x} \leq x<\bar{x}$, where

$$
\pi^{*}=-\frac{\theta}{\sigma} \frac{V^{\prime}(x)}{V^{\prime \prime}(x)}, \quad u_{L}^{\prime}\left(c^{*}\right)=V^{\prime}(x)
$$

are also obtained from the first order conditions (FOCs). Here $h$ is a constant defined in (3.8).

In order to solve HJB equation (3.9), we consider the optimal consumption $c$ as a function of the agent's wealth $x$ by writing $c=C(x)$, and suppose that $C(\cdot)$ has an inverse function $X(\cdot)$. Then we have

$$
V^{\prime}(x)=(\alpha+h)^{\frac{1-\gamma-\rho}{\rho}} C(x)^{-\gamma} \quad \text { and } \quad V^{\prime \prime}(x)=-\gamma(\alpha+h)^{\frac{1-\gamma-\rho}{\rho}} \frac{C(x)^{-\gamma-1}}{X^{\prime}(c)}
$$

and we can rewrite HJB equation (3.9) as follows:

$$
\beta V(X(c))=(\alpha+h)^{\frac{1-\gamma-\rho}{\rho}}\left[\frac{1}{2 \gamma} \theta^{2} c^{1-\gamma} X^{\prime}(c)+r c^{-\gamma} X(c)+w \bar{L} c^{-\gamma}-\tilde{h} c^{1-\gamma}\right]
$$

where $\tilde{h}$ is a constant defined in (3.8). We differentiate (3.13) with respect to $c$ to obtain the following second-order ordinary differential equation (ODE):

$$
\frac{1}{2 \gamma} \theta^{2} c^{2} X^{\prime \prime}(c)+\left(r-\beta+\frac{1-\gamma}{2 \gamma} \theta^{2}\right) c X^{\prime}(c)-r \gamma X(c)-(1-\gamma) \tilde{h} c-w \gamma \bar{L}=0 .
$$

The general solution to ODE (3.14) is given by

$$
X(c)=A_{2} c^{-\gamma n_{2}}-\frac{(1-\gamma) \tilde{h}}{\gamma K} c-\frac{w \bar{L}}{r}
$$

where $A_{2}$ is some constant and $K$ is a constant defined in (3.8) $\left(A_{2}\right.$ will be determined later). Substituting (3.15) into (3.13), we have

$$
\begin{aligned}
V(x) & =(\alpha+h)^{\frac{1-\gamma-\rho}{\rho}}\left\{\frac{r-\frac{1}{2} \theta^{2} n_{2}}{\beta} A_{2} c^{-\gamma n_{2}-\gamma}-\frac{\tilde{h}}{\gamma K} c^{1-\gamma}\right\} \\
& =(\alpha+h)^{\frac{1-\gamma-\rho}{\rho}}\left\{\frac{n_{2}}{p_{2}} A_{2} c^{-\gamma n_{2}-\gamma}-\frac{\tilde{h}}{\gamma K} c^{1-\gamma}\right\} .
\end{aligned}
$$

Similarly, we introduce a function $X_{L}(\cdot)$ such that the agent's wealth $x=X_{L}(c)$ and its inverse function $C_{L}(\cdot)$ such that the optimal consumption $c=C_{L}(x)$ to solve HJB equation 
(3.11). Then we obtain

$$
V^{\prime}(x)=u_{L}^{\prime}\left(C_{L}(x)\right), \quad V^{\prime \prime}(x)=\frac{u_{L}^{\prime \prime}\left(C_{L}(x)\right)}{X_{L}^{\prime}(c)}
$$

and HJB equation (3.11) becomes

$$
\beta V\left(X_{L}(c)\right)=-\frac{1}{2} \theta^{2} \frac{\left(u_{L}^{\prime}(c)\right)^{2}}{u_{L}^{\prime \prime}(c)} X_{L}^{\prime}(c)+\left\{r X_{L}(c)-c+w(\bar{L}-L)\right\} u_{L}^{\prime}(c)+u_{L}(c) .
$$

If we differentiate (3.16) with respect to $c$, then we obtain

$$
\begin{aligned}
\frac{1}{2} \theta^{2} X_{L}^{\prime \prime}(c)= & \left\{\left(r-\beta-\theta^{2}\right) \frac{u_{L}^{\prime \prime}(c)}{u_{L}^{\prime}(c)}+\frac{1}{2} \theta^{2} \frac{u_{L}^{\prime \prime \prime}(c)}{u_{L}^{\prime \prime}(c)}\right\} X_{L}^{\prime}(c)+r\left\{\frac{u_{L}^{\prime \prime}(c)}{u_{L}^{\prime}(c)}\right\}^{2} X_{L}(c) \\
& +\{-c+w(\bar{L}-L)\}\left\{\frac{u_{L}^{\prime \prime}(c)}{u_{L}^{\prime}(c)}\right\}^{2} .
\end{aligned}
$$

The variation of parameters method for ODE (3.17) provides us with a particular solution $X_{L, p}(c)$ to ODE (3.17):

$$
\begin{aligned}
X_{L, p}(c)= & -\frac{2}{\theta^{2}\left(n_{1}-n_{2}\right)}\left\{\frac{\left(u_{L}^{\prime}(c)\right)^{n_{1}}}{n_{1}} \int_{0}^{c} \frac{d y}{\left(u_{L}^{\prime}(y)\right)^{n_{1}}}+\frac{\left(u_{L}^{\prime}(c)\right)^{n_{2}}}{n_{2}} \int_{c}^{\infty} \frac{d y}{\left(u_{L}^{\prime}(y)\right)^{n_{2}}}\right\} \\
& +\frac{c}{r}-\frac{w(\bar{L}-L)}{r} .
\end{aligned}
$$

The general solution to ODE (3.17) is then given by

$$
X_{L}(c)=B_{1}\left(u_{L}^{\prime}(c)\right)^{n_{1}}+B_{2}\left(u_{L}^{\prime}(c)\right)^{n_{2}}+X_{L, p}(c)
$$

where $B_{1}$ and $B_{2}$ are some constants to be determined later. Plugging (3.18) into (3.16) gives rise to

$$
V(x)=\frac{n_{1}}{p_{1}} B_{1}\left(u_{L}^{\prime}(c)\right)^{p_{1}}+\frac{n_{2}}{p_{2}} B_{2}\left(u_{L}^{\prime}(c)\right)^{p_{2}}+J_{L}(c),
$$

where

$$
J_{L}(c)=\frac{u_{L}(c)}{\beta}-\frac{2}{\theta^{2}\left(p_{1}-p_{2}\right)}\left\{\frac{\left(u_{L}^{\prime}(c)\right)^{p_{1}}}{p_{1}} \int_{0}^{c} \frac{d y}{\left(u_{L}^{\prime}(y)\right)^{n_{1}}}+\frac{\left(u_{L}^{\prime}(c)\right)^{p_{2}}}{p_{2}} \int_{c}^{\infty} \frac{d y}{\left(u_{L}^{\prime}(y)\right)^{n_{2}}}\right\} .
$$

After retirement, we derive the value function $V(x)=U(x)=\bar{J}(\bar{C}(x))$ from Karatzas et al. [10], where a function $\bar{X}(\cdot)$ and its inverse function $\bar{C}(\cdot)$ are given such that the agent's wealth $x=\bar{X}(c)$ and the optimal consumption $c=\bar{C}(x)$, and $\bar{X}(c)$ and $\bar{J}(c)$ are defined in (3.7).

Now we are going to determine the coefficients $A_{1}, B_{1}, B_{2}$ and the wealth boundaries $\tilde{x}$ and $\bar{x}$. First, we use the continuity of $V^{\prime}(x)$ at $x=\tilde{x}$. We then have

$$
V^{\prime}(\tilde{x})=(\alpha+h)^{\frac{1-\gamma-\rho}{\rho}} \tilde{c}^{-\gamma}=\tilde{c}^{\rho-1}\left\{\alpha \tilde{c}^{\rho}+(1-\alpha) L^{\rho}\right\}^{\frac{1-\gamma-\rho}{\rho}},
$$


from which we obtain the relation

$$
\tilde{c}=\left(\frac{w \alpha}{1-\alpha}\right)^{\frac{1}{1-\rho}} L,
$$

where $\tilde{c}$ is the optimal consumption rate corresponding to $\tilde{x}$, that is, $\tilde{x}=X(\tilde{c})$. Also the continuity of $V(x)$ at $x=\tilde{x}$ implies

$$
\begin{aligned}
V(\tilde{x}) & =(\alpha+h)^{\frac{1-\gamma-\rho}{\rho}}\left\{\frac{n_{2}}{p_{2}} \tilde{c}^{-\gamma n_{2}-\gamma} A_{2}-\frac{\tilde{h}}{\gamma K} \tilde{c}^{1-\gamma}\right\} \\
& =\frac{n_{1}}{p_{1}}\left(u_{L}^{\prime}(\tilde{c})\right)^{p_{1}} B_{1}+\frac{n_{2}}{p_{2}}\left(u_{L}^{\prime}(\tilde{c})\right)^{p_{2}} B_{2}+J_{L}(\tilde{c}) .
\end{aligned}
$$

By (3.15) and (3.18), we obtain

$$
\begin{aligned}
\tilde{x} & =\tilde{c}^{-\gamma n_{2}} A_{2}-\frac{(1-\gamma) \tilde{h}}{\gamma K} \tilde{c}-\frac{w \bar{L}}{r} \\
& =\left(u_{L}^{\prime}(\tilde{c})\right)^{n_{1}} B_{1}+\left(u_{L}^{\prime}(\tilde{c})\right)^{n_{2}} B_{2}+X_{L, p}(\tilde{c}) .
\end{aligned}
$$

Combining (3.20), (3.21), and the fact $u_{L}^{\prime}(\tilde{c})=(\alpha+h)^{\frac{1-\gamma-\rho}{\rho}} \tilde{c}^{-\gamma}$, we obtain

$$
\begin{aligned}
B_{1}= & \frac{\tilde{h}}{\left(\frac{n_{2}}{p_{2}}-\frac{n_{1}}{p_{1}}\right) \gamma K}\left\{1-\frac{n_{2}}{p_{2}}(1-\gamma)\right\} \tilde{c}\left(u_{L}^{\prime}(\tilde{c})\right)^{-n_{1}} \\
& -\frac{n_{2}}{\left(\frac{n_{2}}{p_{2}}-\frac{n_{1}}{p_{1}}\right) p_{2}}\left\{\frac{w \bar{L}}{r}+X_{L, p}(\tilde{c})\right\}\left(u_{L}^{\prime}(\tilde{c})\right)^{-n_{1}}+\frac{J_{L}(\tilde{c})}{\frac{n_{2}}{p_{2}}-\frac{n_{1}}{p_{1}}}\left(u_{L}^{\prime}(\tilde{c})\right)^{-p_{1}} .
\end{aligned}
$$

Second, we use the smooth-pasting condition of $V(x)$ at $x=\bar{x}$ to determine $\bar{x}$ and $\bar{c}$, where $\bar{c}$ is the optimal consumption rate corresponding to $\bar{x}$. Since the leisure rate is discontinuous at $x=\bar{x}$, we conjecture that there are $\bar{c}_{L}$ and $\bar{c}\left(\bar{c}_{L} \neq \bar{c}\right)$ such that

$$
\bar{x}=X_{L}\left(\bar{c}_{L}\right)=\bar{X}(\bar{c}) .
$$

Thus we have

$$
\bar{x}=B_{1}\left(u_{L}^{\prime}\left(\bar{c}_{L}\right)\right)^{n_{1}}+B_{2}\left(u_{L}^{\prime}\left(\bar{c}_{L}\right)\right)^{n_{2}}+X_{L, p}\left(\bar{c}_{L}\right)=\bar{X}(\bar{c})
$$

and

$$
V(\bar{x})=\frac{n_{1}}{p_{1}} B_{1}\left(u_{L}^{\prime}\left(\bar{c}_{L}\right)\right)^{p_{1}}+\frac{n_{2}}{p_{2}} B_{2}\left(u_{L}^{\prime}\left(\bar{c}_{L}\right)\right)^{p_{2}}+J_{L}\left(\bar{c}_{L}\right)=\bar{J}(\bar{c})
$$

from (3.18) and (3.19), respectively. From (3.23) and (3.24), we have

$$
\left(\frac{n_{2}}{p_{2}}-\frac{n_{1}}{p_{1}}\right) B_{1}\left(u_{L}^{\prime}\left(\bar{c}_{L}\right)\right)^{p_{1}}+\frac{n_{2}}{p_{2}} u_{L}^{\prime}\left(\bar{c}_{L}\right)\left\{X_{L, p}\left(\bar{c}_{L}\right)-\bar{X}(\bar{c})\right\}=\left\{J_{L}\left(\bar{c}_{L}\right)-\bar{J}(\bar{c})\right\} .
$$

The $C^{1}$-condition of $V(x)$ at $x=\bar{x}$ and FOCs (3.12) give us

$$
V^{\prime}(\bar{x})=u_{L}^{\prime}\left(\bar{c}_{L}\right)=\frac{\bar{J}^{\prime}(\bar{c})}{\bar{X}^{\prime}(\bar{c})}
$$


and, consequently,

$$
\bar{c}_{L}=I_{L}\left(\frac{\bar{J}^{\prime}(\bar{c})}{\bar{X}^{\prime}(\bar{c})}\right)
$$

where $I_{L}(\cdot)$ is the inverse function of $u_{L}^{\prime}(\cdot)$. Substituting (3.26) into (3.25) implies

$$
\begin{aligned}
& \left(\frac{n_{2}}{p_{2}}-\frac{n_{1}}{p_{1}}\right) B_{1}\left(\frac{\bar{J}^{\prime}(\bar{c})}{\bar{X}^{\prime}(\bar{c})}\right)^{p_{1}}+\frac{n_{2}}{p_{2}} \frac{\bar{J}^{\prime}(\bar{c})}{\bar{X}^{\prime}(\bar{c})}\left\{X_{L, p}\left(I_{L}\left(\frac{\bar{J}^{\prime}(\bar{c})}{\bar{X}^{\prime}(\bar{c})}\right)\right)-\bar{X}(\bar{c})\right\} \\
& \quad-J_{L}\left(I_{L}\left(\frac{\bar{J}^{\prime}(\bar{c})}{\bar{X}^{\prime}(\bar{c})}\right)\right)+\bar{J}(\bar{c})=0 .
\end{aligned}
$$

If $\bar{c}$ is obtained from (3.27), then $\bar{c}_{L}$ is also obtained from (3.26). Thus we can get $\bar{x}$ from (3.22). From (3.23) and (3.21), we also derive

$$
B_{2}=-B_{1}\left(u_{L}^{\prime}\left(\bar{c}_{L}\right)\right)^{n_{1}-n_{2}}+\left\{\bar{x}-X_{L, p}\left(\bar{c}_{L}\right)\right\}\left(u_{L}^{\prime}\left(\bar{c}_{L}\right)\right)^{-n_{2}}
$$

and

$$
A_{2}=\tilde{c}^{\gamma n_{2}} X_{L}(\tilde{c})+\frac{(1-\gamma) \tilde{h}}{\gamma K} \tilde{c}^{\gamma n_{2}+1}+\frac{w \bar{L}}{r} \tilde{c}^{\gamma n_{2}} .
$$

FOCs (3.10) and (3.12) give us the following optimal strategies.

Theorem 3.3 The optimal strategies $\left(\mathbf{c}^{*}, \mathbf{l}^{*}, \pi^{*}, \tau^{*}\right)$ are given by

$$
\begin{gathered}
c_{t}^{*}=\left\{\begin{array}{ll}
C\left(x_{t}\right) & \text { for }-\frac{w \bar{L}}{r}<x_{t}<\tilde{x}, \\
C_{L}\left(x_{t}\right) & \text { for } \tilde{x} \leq x_{t}<\bar{x}, \\
\bar{C}\left(x_{t}\right) & \text { for } x_{t} \geq \bar{x},
\end{array} \quad l_{t}^{*}= \begin{cases}\left(\frac{1-\alpha}{w \alpha}\right)^{\frac{1}{1-\rho}} C\left(x_{t}\right) & \text { for }-\frac{w \bar{L}}{r}<x_{t}<\tilde{x}, \\
L & \text { for } \tilde{x} \leq x_{t}<\bar{x}, \\
\bar{L} & \text { for } x_{t} \geq \bar{x},\end{cases} \right. \\
\pi_{t}^{*}= \begin{cases}\frac{\theta}{\sigma \gamma}\left\{-\gamma n_{2} A_{2}\left(C\left(x_{t}\right)\right)^{-\gamma n_{2}}-\frac{(1-\gamma) \tilde{h}}{\gamma K} C\left(x_{t}\right)\right\} & \text { for }-\frac{w \bar{L}}{r}<x_{t}<\tilde{x}, \\
\frac{\theta}{\sigma} \frac{\alpha\left(C_{L}\left(x_{t}\right)\right)^{\rho}+(1-\alpha) L^{\rho}}{\alpha \gamma\left(C_{L}\left(x_{t}\right)\right)^{\rho}(1-\alpha)\left(1^{-\rho}\right) L^{\rho}} C_{L}\left(x_{t}\right) X_{L}^{\prime}\left(C_{L}\left(x_{t}\right)\right) & \text { for } \tilde{x} \leq x_{t}<\bar{x}, \\
\frac{\theta}{\sigma} \frac{\alpha\left(\bar{x}\left(x_{t}\right) \rho^{\rho}+(1-\alpha) L^{\rho}\right.}{\alpha \gamma\left(\bar{C}\left(x_{t}\right)\right)^{\rho}+(1-\alpha)(1-\rho) \bar{L}^{\rho}} \bar{C}\left(x_{t}\right) \bar{X}^{\prime}\left(\bar{C}\left(x_{t}\right)\right) & \text { for } x_{t} \geq \bar{x},\end{cases}
\end{gathered}
$$

and

$$
\tau^{*}=\inf \left\{t \geq 0: x_{t} \geq \bar{x}\right\}
$$

Remark 3.2 The optimal strategies $\left(\mathbf{c}^{*}, \mathbf{I}^{*}, \boldsymbol{\pi}^{*}, \tau^{*}\right)$ in Theorem 3.3 are the same as those in Theorem 5.3 from Choi et al. [9]. Although it is difficult to obtain an explicit transformation between them, simple but tedious calculations show that they are equivalent.

\section{Numerical examples and Cobb-Douglas utility}

In this section, we present numerical examples and a limiting case $\rho \rightarrow 0$, that is, the elasticity of substitution goes to 1 . In this case, our CES utility function becomes a CobbDouglas utility function.

In Figure 1, we see that there exists a discontinuity of the optimal consumption at the threshold wealth level $x=\bar{x}$. This result is similar to Figure 5.1 in Choi et al. [9] which was obtained in the limiting case $\rho \rightarrow 0$. 


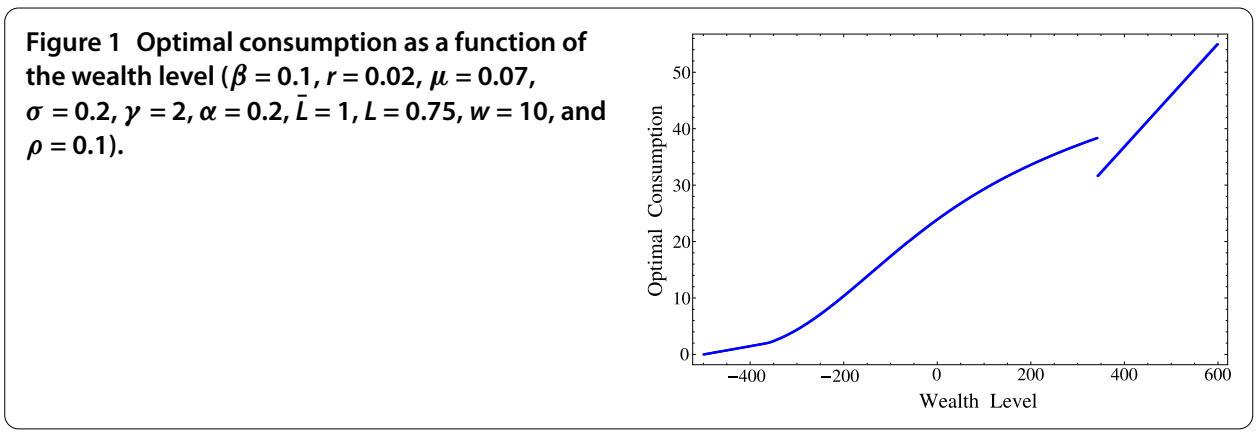

Figure 2 Retirement wealth level as a function of the elasticity of substitution $1 /(1-\rho)(\beta=0.1$, $r=0.02, \mu=0.07, \sigma=0.2, \alpha=0.2, \bar{L}=1, L=0.75$, and $w=10)$.

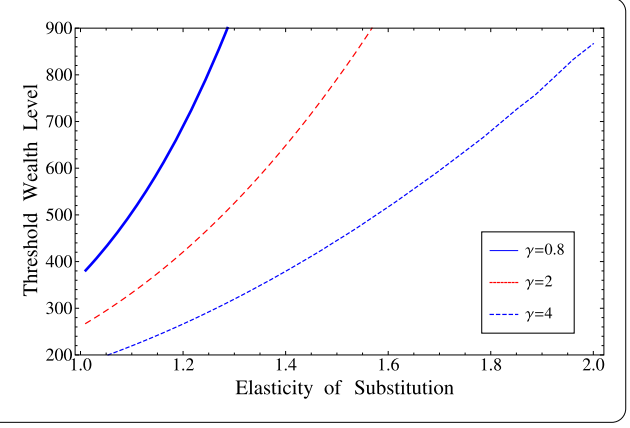

In Figure 2, we observe that the critical wealth level $\bar{x}$ is an increasing function with respect to the elasticity of substitution between consumption and leisure, $1 /(1-\rho)$. In this model, retirement incentive is derived from a large leisure rate while not working. Before retirement, an economic agent's leisure rate is upper limited by $L$, and when the leisure rate at which the economic agent optimally enjoys reaches $L$, she is forced to reduce leisure and to increase consumption to retain the utility. In this situation, an economic agent with a large elasticity of substitution between consumption and leisure may consume more and enjoy higher utility than an economic agent with a small elasticity of substitution. So the former has a larger incentive to stay working and a higher threshold retirement wealth level than the latter and tends to delay retirement.

Let us investigate a limiting case. A Cobb-Douglas utility function is defined by

$$
u_{\mathrm{CD}}(c, l) \triangleq \frac{1}{\alpha} \cdot \frac{\left(c^{\alpha} l^{1-\alpha}\right)^{1-\gamma}}{1-\gamma}, \quad 0<\alpha<1, \gamma>0 \text { and } \gamma \neq 1
$$

which is a special case of a CES utility function given in (3.1) due to the fact that

$$
\lim _{\rho \rightarrow 0} \frac{1}{\alpha} \cdot \frac{\left\{\alpha c^{\rho}+(1-\alpha) l^{\rho}\right\}^{\frac{1-\gamma}{\rho}}}{1-\gamma}=\frac{1}{\alpha} \cdot \frac{\left(c^{\alpha} l^{1-\alpha}\right)^{1-\gamma}}{1-\gamma}
$$

from L'Hôpital's rule. Also note that

$$
\lim _{\rho \rightarrow 0} h=1-\alpha, \quad \lim _{\rho \rightarrow 0} \tilde{h}=-\frac{\gamma}{\alpha(1-\gamma)}, \quad \lim _{\rho \rightarrow 0}(\alpha+h)^{\frac{1-\gamma-\rho}{\rho}}=\left(\frac{w \alpha}{1-\alpha}\right)^{-\left(\gamma_{1}-\gamma\right)},
$$

and

$$
\lim _{\rho \rightarrow 0}(\alpha+h)^{\frac{1-\gamma-\rho}{\rho}} \tilde{c}^{-\gamma}=\tilde{c}_{\mathrm{s}}^{-\gamma_{1}} L^{\gamma_{1}-\gamma}
$$


where

$$
\gamma_{1} \triangleq 1-\alpha(1-\gamma), \quad \tilde{c}_{s}=\left(\frac{w \alpha}{1-\alpha}\right) L .
$$

Using the relation

$$
\frac{r-\frac{1}{2} \theta^{2} n_{1,2}}{\beta}=\frac{n_{1,2}}{p_{1,2}}
$$

we also derive

$$
\lim _{\rho \rightarrow 0} A_{2}=A_{2, \mathrm{~s}}, \quad \lim _{\rho \rightarrow 0} B_{1}=L^{\left(\gamma-\gamma_{1}\right) n_{1}} B_{1, \mathrm{~s}}, \quad \lim _{\rho \rightarrow 0} B_{2}=L^{\left(\gamma-\gamma_{1}\right) n_{2}} B_{2, \mathrm{~s}},
$$

where $A_{2, \mathrm{~s}}, B_{1, \mathrm{~s}}, B_{2, \mathrm{~s}}$ are the coefficients $A_{2}, B_{1}, B_{2}$ of Theorem 3.1 in Koo et al. [6], respectively. Therefore, we see that the value function and optimal strategies of the agent in this paper coincide with those of Koo et al. [6].

\section{Conclusion}

With the dynamic programming method, we have provided some methodological contributions to the previous research, Choi et al. [9] which solved an optimal portfolio, consumption-leisure and retirement choice problem for an infinitely lived economic agent with a CES utility function. Through some illustrative numerical examples, we see that the threshold retirement wealth level increases with the elasticity of substitution. The solutions to a limiting case when the elasticity of substitution goes to 1 become those of the optimization problem with a Cobb-Douglas utility function.

\section{Competing interests}

The authors declare that they have no competing interests.

\section{Authors' contributions}

All authors contributed equally to the writing of this paper.

\section{Author details}

${ }^{1}$ Research Institute of Finance \& Risk Management, POSTECH, Pohang, 37673, Republic of Korea. ${ }^{2}$ Department of Mathematics, Sookmyung Women's University, Seoul, 04310, Republic of Korea.

\section{Acknowledgements}

The corresponding author (YH Shin) gratefully acknowledges the support of Sookmyung Women's University Research Grants 2013 (1-1303-0272)

Received: 12 August 2015 Accepted: 24 September 2015 Published online: 06 October 2015

\section{References}

1. Bodie, Z, Merton, RC, Samuelson, WF: Labor supply flexibility and portfolio choice in a life cycle model. J. Econ. Dyn. Control 16, 427-449 (1992)

2. Merton, RC: Lifetime portfolio selection under uncertainty: the continuous-time case. Rev. Econ. Stat. 51, 247-257 (1969)

3. Merton, RC: Optimum consumption and portfolio rules in a continuous-time model. J. Econ. Theory 3, 373-413 (1971)

4. Farhi, E, Panageas, S: Saving and investing for early retirement: a theoretical analysis. J. Financ. Econ. 83, 87-121 (2007)

5. Shin, $\mathrm{YH}$ : Voluntary retirement and portfolio selection: dynamic programming approaches. Appl. Math. Lett. 25, 1087-1093 (2012)

6. Koo, JL, Koo, BL, Shin, YH: An optimal investment, consumption, leisure, and voluntary retirement problem with Cobb-Douglas utility: dynamic programming approaches. Appl. Math. Lett. 26, 481-486 (2013)

7. Dybvig, PH, Liu, H: Lifetime consumption and investment: retirement and constrained borrowing. J. Econ. Theory 145, 885-907 (2010) 
8. Barucci, E, Marazzina, D: Optimal investment, stochastic labor income and retirement. Appl. Math. Comput. 213, 5588-5604 (2012)

9. Choi, KJ, Shim, G, Shin, YH: Optimal portfolio, consumption-leisure and retirement choice problem with CES utility. Math. Finance 18, 445-472 (2008)

10. Karatzas, I, Lehoczky, JP, Sethi, SP, Shreve, SE: Explicit solution of a general consumption/investment problem. Math. Oper. Res. 11, 261-294 (1986)

11. Choi, KJ, Shim, G: Disutility, optimal retirement, and portfolio selection. Math. Finance 16, $443-467$ (2006)

12. Dybvig, $\mathrm{PH}, \mathrm{Liu}, \mathrm{H}$ : Verification theorems for models of optimal consumption and investment with retirement and constrained borrowing. Math. Oper. Res. 36, 620-635 (2011)

Submit your manuscript to a SpringerOpen ${ }^{\odot}$ journal and benefit from:

- Convenient online submission

- Rigorous peer review

- Immediate publication on acceptance

- Open access: articles freely available online

- High visibility within the field

Retaining the copyright to your article

Submit your next manuscript at $\gg$ springeropen.com 